\title{
A Decade of Step and Flash Imprint Lithography
}

\author{
C. Grant Willson \\ Department of Chemical Engineering \\ The University of Texas, \\ Austin, Texas 78712, USA
}

\begin{abstract}
Step and Flash Imprint Lithography (SFIL) was first reported ten years ago. The technology has advanced in many ways during the last decade. There are sophisticated, imprint tools available from commercial suppliers and high resolution templates can be purchased from several vendors. The materials and the process have evolved and matured to enable printing of sub $10 \mathrm{~nm}$ structures, inverse tone printing, printing on both sides of disk drive substrates, printing in three dimensions to enable efficient production of CMOS wiring levels and printing of functional materials tailored to enable production of photonic crystals, insulators, etc. While great progress has been made in many areas, there remain concerns among some about defectivity and the throughput of the process.
\end{abstract}

Keyword: Step and Flash Imprint Lithography, BEOL, nano-imprint, templates

\section{Introduction}

Step and Flash Imprint Lithography (SFIL) was announced publically ten years ago [1]. The early work was done on a tool that was installed at the University of Texas. This tool, which was constructed on the body of an Ultratech ${ }^{\circledR}$ deep UV stepper embodied many of the important design features of current tools and quickly produced very high resolution images. A picture of the first SFIL tool is provided in Figure 1.

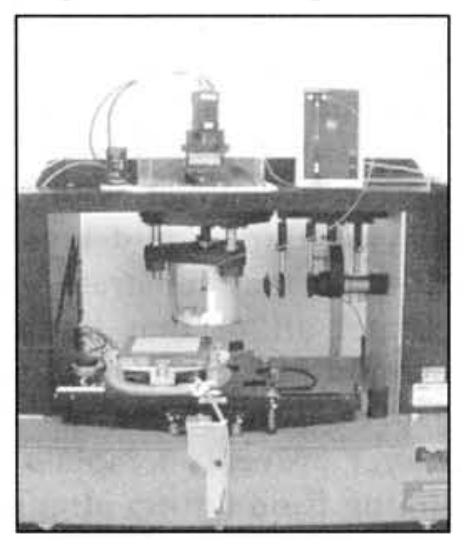

Figure 1: The first SFIL tool installed at the University of Texas at Austin
The SFIL process patents and the basic tool design were licensed to Molecular Imprints, Inc who have evolved the design to the point where they are supplying a range of tools, including the Imprio 300 shown in Figure 2 below, which has been installed at Sematech in Albany, New York and in the development facilities of several semiconductor manufacturers. This tool is capable of sub-20nm overlay and has a throughput of approximately 5, 300 $\mathrm{mm}$ wafers per hour.

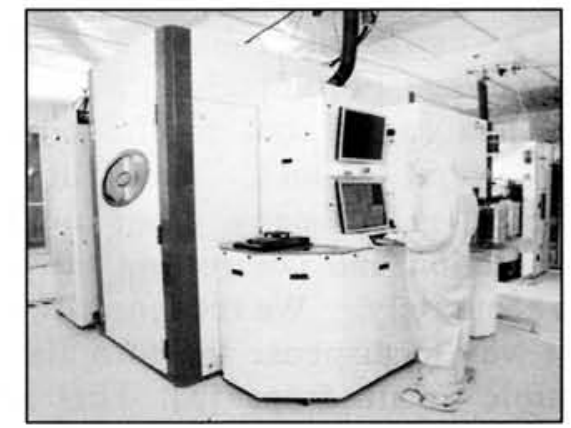

Figure 2: Molecular Imprints Imprio 300 
These tools are being exercised to explore their viability for CMOS manufacturing and to produce samples of very high resolution patterns and parts for early device learning. The evolution of this hardware and the associated processes and infrastructure are the subjects of this paper.

\section{Results and Discussion}

Very early in the SFIL work it was recognized that there were several important engineering challenges that had to be addressed. Failure to solve any one of these problems would have been a "show stopper".

The important issue was template placement and removal. Clearly it is necessary to bring the patterned SFIL template into contact with the liquid monomer formulation such that the bottom surface of the template is very nearly parallel to the substrate. It must then come into perfect parallelism before the photopolymerization step and after the formulation is rendered solid by the exposure, the template must be removed without rotation around the $Z$ axis or translation in the $X . Y$ plane even to the extend of a few nanometers or the patterns that were generated will be sheared off of the substrate. Accomplishment of this was achieved by a very simple but clever flexure joint design that was done by Prof. S.V. Sreenivasan [2]. This design has evolved and is now much more sophisticated, but the basic principles described in reference 2 still apply.

The second issue that needed to be resolved was the filling of the template and control of the residual layer. The earliest SFIL patterns were invariably generated with a great deal of fluid extrusion beyond the limits of the template and with thick and irregular residual layers. The extrusion problem at first looked quite daunting. Fortunately, the solution to this problem came from careful engineering of the wafer chuck, and other tool components, using many drops to dispense the formulation and controlling the dispense volume accurately. We recognized early that the best way to dispense the formulation was in multiple small drops [3]. This approach offers several important advantages over spin coating or other methods of fluid dispense.

First, it provides a means to distribute the liquid in a pattern that matches the local variations in pattern density on the template such that a minimal amount of fluid flow is required to achieve fill. [3] Second, it means that the template is pulled down into the fluid by capillary forces that derive from spreading of the droplets until they coalesce into a continuous film $[3,4]$. Thirdly, it is fortuitous that once the drops do coalesce, the force required to make the next movement in the $Z$ direction requires moving the liquid through a very thin sheet $(\sim 15-20 \mathrm{~nm})$ and that requires a large force and a long time. So, if the proper amount of fluid is dispensed as small drops and dispensed in areas where it is needed to fill the template relief patterns, the template is pulled into position. The fill goes to the very edge of the mesa (see Figure 3 ) and it stops there "automatically". If the coalescence occurs to produce a thin residual layer, which is the design point, then further attempts to reduce the residual layer thickness by movement in $Z$ require very high forces and long times. This allows placing the die in very close proximity to one another without fear of extrusion of the formulation. The mesa on which the high resolution patterns are etched is 15 microns thicker than the fused silica substrate.

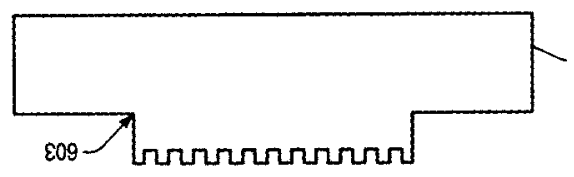

Figure 3. The SFIL template design.

Many ask how much force is required to bring the template into contact with the wafer. The answer to that question is that it does not require application of any force, the template is pulled down and filled my capillary forces and unless there is some catastrophic error, it NEVER comes into contact with the wafer. There is always a thin, liquid layer between the template and the substrate. It is not possible to expel all of that liquid because doing so would require pushing liquid through a gap of ever decreasing thickness. That liquid layer prevents template wear and acts as a damping fluid during alignment.

It is now possible to control the thickness of the residual layer to nominally $15-20 \mathrm{~nm}$ thickness with a variance of $1.5 \mathrm{~nm} 3 \sigma$ over a 
$300 \mathrm{~mm}$ wafer.

The next problem that had to be solved was template release. No applied force is required to fill the template, but force is required to remove it after the photo-polymerization of the monomer formulation, which is often referred to as "resist". Separation requires tenacious adhesion of the resist to the substrate and adhesive failure at the template-resist interface. If the resist undergoes cohesive failure, the fidelity of the pattern transfer is lost and the template is fouled. The templates for SFIL are fabricated from the standard, fused silica blanks used to make photo-masks. A detailed study of the treatment of the $\mathrm{SiO}_{2}$ surface was undertaken and methods were published for producing a robust self assembled monolayer (SAM) of perfluoroalkylsilanes on the surface [6]. A number of studies including efforts in our laboratories [7] have shown that regardless of the care with which these layers are assembled, they are slowly removed by the SFIL process. $[7,8]$

Treatment of the template surface may be necessary for good release, but it is not sufficient. It is not duarable. We therefore auditioned several additives to the resist formulation that serve to minimize the release force and extend the durability of the template. We accomplished this by addition of certain fluorinated surfactants to the formulation [6]. Over 10,000 imprints have been made with the same template with no statistically significant indication of wear and recently, more than 10,000 imprints with a single template were reported without any cleaning. [8] While there is still work to be done to improve template release, it does not appear to be a show stopper level problem.

The availability of SFIL templates was a challenge for the mask industry because the features are $1 \mathrm{X}$, the same size as the printed features, while for typical photomasks, the mask image is $4 \mathrm{X}$ the final image. This factor of 4 increase in the demand for the resolution from the electron beam mask writers was a challenge. Templates with very nice resolution test patterns at $50 \mathrm{~nm}$ and smaller were available early in the program from Gaussian beam writing tools, but the writing time for a full scale device template on these machines is too long to be practical.
Recently commercial suppliers have provided sub-50nm templates written with shaped beam tools. (Figure 4) The write time for these templates is equal to or shorter than that required to write the same level in a phase shift optical mask. [9] This is a very encouraging, but there are remaining problems to solve with the template infrastructure. The first is inspection of the templates. There is no inspection tool available that can inspect the final template. Scanning electron microscopy on quartz is difficult due to charging effects. At this time, it seems that template inspection will have to be done on imprinted images rather than on the template itself.

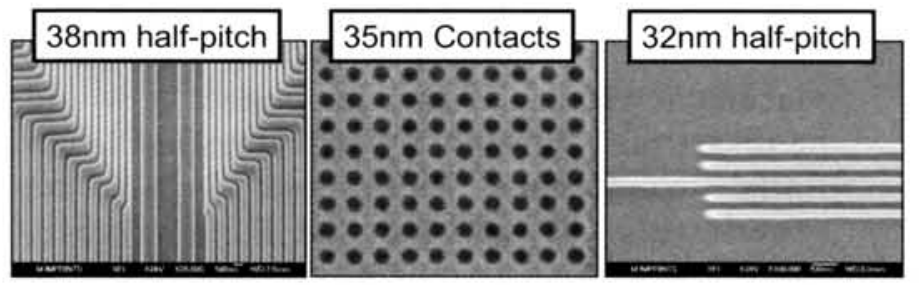

Figure 4. Imprints from templates written with shaped beam mask writers.

The other issue with template manufacturing is that the production of the mesa and dicing of the blank are not standard unit processes in today's mask houses. These are not standard processes used to make photomasks. Hence, these steps are being done outside of the very clean process areas within commercial mask houses. The solution to this problem is either to change the format of the template to a six inch square plate so that no new fixtures are required and there is no dicing step, or to install these processes in the mask lines. It is unclear which path is most likely at this writing, but two clear solutions to the problem do exist.

Alignment was also a challenge area. In particular, SFIL must be compatible in a mix and match process with $193 \mathrm{~nm}$ optical exposure tools. This means not only must the SFIL tool succeed in $\mathrm{X}, \mathrm{Y}$ and Theta alignment; it must be able to do magnification correction. The details of the Moire fringe alignment system that has been published will not be covered in detail here, but those are available in the literature [10]. The use of controlled compression of the template to achieve magnification correction and to 
correct for most field shape distortions has been demonstrated to below $20 \mathrm{~nm} 3 \sigma$. $[10,11,12]$. Like in all other lithography technologies, alignment will always be a challenge but no unique SFIL issues have emerged that limit the accuracy and precision of alignment. Improvement will require continued, careful and detailed engineering work, but no limiting problems have emerged.

Another area of serious early concern was pattern placement error due to shrinkage of the resist formulation. The concern was that the patterns sit on a thin polymer film, the residual layer and the volumetric shrinkage of the formulation can be more than $15 \%$ upon exposure. If a large pad is situated next to a line, the shrinkage of the pad could pull the line toward it, causing a pattern dependent placement error. If that happened, it would be disastrous. Fortunately, this does not occur. Early simulations [13] and subsequent experiments [14] showed that the shrinkage, for all practical purposes occurs in the $Z$ direction, perpendicular to the plane of the film. It causes small and predictable changes in wall slope, but does not affect pattern placement.

Defectivity and throughput are the other issues most commonly raised as concerns. The throughput of the process has a unique, SFIL component that does not exist in photolithography. That is, the filling of the template. All other steps, apply, align, expose are common to both SFIL and photolithography. The fill process has been studied in detail [4]. The secret to rapid filling is to dispense the resist in a pattern that is matched to the pattern density of the template so that movement of the liquid is minimized. Large scale redistribution of the liquid through the residual layer is slow for the reasons explained earlier. Hence, the dispense is done by studying the template design and dispensing more liquid to areas where there are dense patterns and less liquid in areas where pattern density is sparse. On the commercial tools, this is done through use of a high resolution inkjet dispense system. Further improvement can be achieved by changing the shape of the template from convex to flat as it makes contact with the fluid [8]. The most advanced exposure tool that is available has a throughput of approximately $5300 \mathrm{~mm}$ wafers/ hour. It is faster that the $\alpha$-EUV tools but not fast enough. A pathway to $20 \mathrm{wafers} / \mathrm{hr}$ has been disclosed [8] and it is possible to multiplex or gang the imprint heads in one enclosure. This has been demonstrated in a tool designed to print hard disk drive (HDD) substrates, which has a throughput or approximately 180 disks/hour printed on both sides [8]. SFIL is not likely to produce 100 wafers/hour with a single head until or unless some invention occurs, but that sort of throughput is not required to realize large savings in cost of ownership $[15,16]$.

Defects in SFIL are a major concern to some. The process looks like contact printing on cursory inspection and it had no pellicle. Contact printing was abandoned in the early 1980 's due to defectivity. Why would anyone propose to go back to that sort of process? First, we did a bad job of contact printing. We tried to use chromium on glass mask in contact with a resist that was formulated from rubber based glue. It is not surprising that the resist tended to stick to the mask from time to time. Since that time, scientists have learned to tailor the surface energy of materials and control these things. Postage stamps are easily peeled from "treated" paper but stick irreversibly to untreated envelopes. Adaptation of this sort of release layer chemistry to contact printing produces a low defectivity process. [17]. In the same way, engineering the surface energy at the interfaces in SFIL makes it very different from the ancient contact printing processes.

There is no pellicle, but there is an interesting process of self cleaning that is inherent in the SFIL process. In most cases, if there is a particle on the template, it is removed by trapping it in the photopolymerized resist. In that case, one die is lost but the particle does not print ever more as it would on a photomask without a pellicle. Printing is a good way to clean the template!

There has not yet been published any definitive SFIL defectivity experiment. We have achieved excellent electrical test data on via chains [18] and Molecular Imprints, Inc. has published encouraging trends in reduction of larger defects [8], but a rigorous electrical test yield based study has not yet been 
published. Hopefully these data will emerge soon and the question about serious defects will be answered. The defectivity of this process may well be higher than that of photolithography. We will find out soon.

SFIL was originally envisioned as a low cost, high resolution alternative to photolithography and EUV lithography for CMOS production. In the time that has passed since the original work, a number of non-CMOS applications have emerged for which this patterning technology seems ideally suited. The first of these is pattering of the magnetic media for hard disk drives. The hard disk drive industry hopes to provide a next generation of drives with patterned media. Patterning the media provides a means for reducing the signal to noise ratio for the technology and can also improve the temporal stability of stored data. [19] The resolution requirements for patterning media are very aggressive. There are plans to pattern at $20 \mathrm{~nm}$ and below. The templates for this process are being written by advanced electron beam patterning techniques on rotating stages. The process is advancing rapidly as shown in Figure 5 and represents some of the most aggressive lithography in the world. Patterning of media is expected to enable information storage at $1 \mathrm{~T}-\mathrm{bit} / \mathrm{in}^{2}$

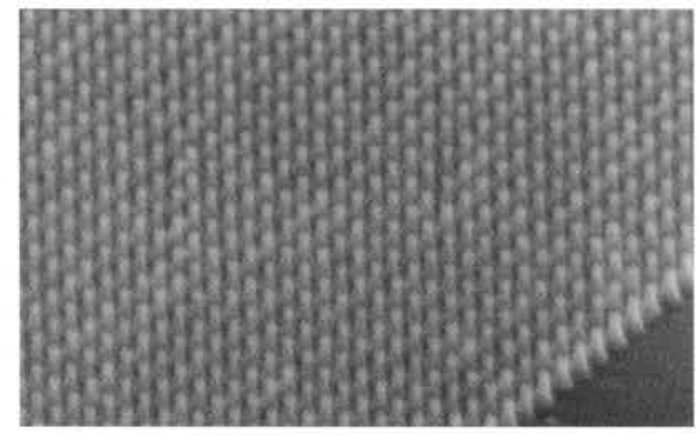

Figure 5. Sub-20nm patterning on a hard disk substrate.

Another interesting non-CMOS application involves patterning material with a controlled index of refraction to form photonic crystals that are used to improve light extraction and control directionality in high brightness light emitting diodes (LEDs). Figure 6 shows sub-100nm photonic crystals made up of tiny lenses arranged in complex patterns.

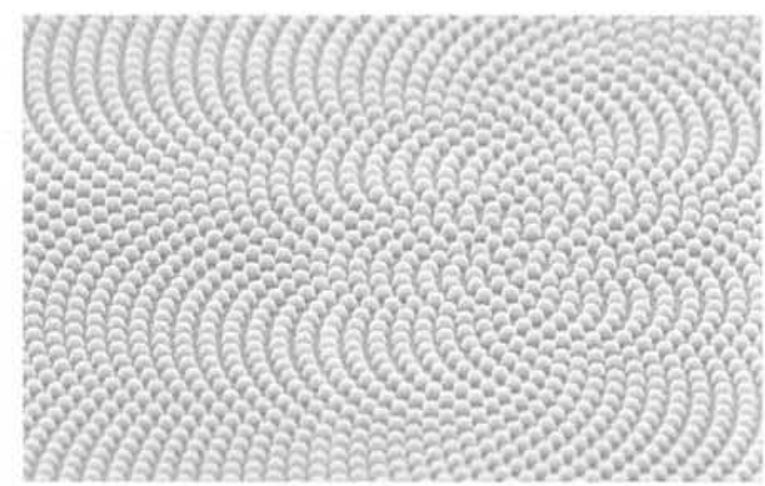

Figure 6. Sub 100nm photonic crystal pattern for application in LED technology [20].

Another application that exploits the ability of SFIL to define structures not only in the $X, Y$ plane of the substrate, but along the $Z$ axis as well is definition of copper interconnects on CMOS devices. In this application it is the ability to simultaneously pattern vias and a wires in a single step that makes the process so interesting. SFIL offers the opportunity to remove many steps from the dual damascene process used in the "Back End of the Line" (BEOL) [18]. A special, multilevel template is employed in the process. The first of these were laboratory curiosities [21,22], but they are now commercially available. An atomic force microscopy image of a multi layer template purchased from Toppan, Inc. is shown in Figure 7 below.

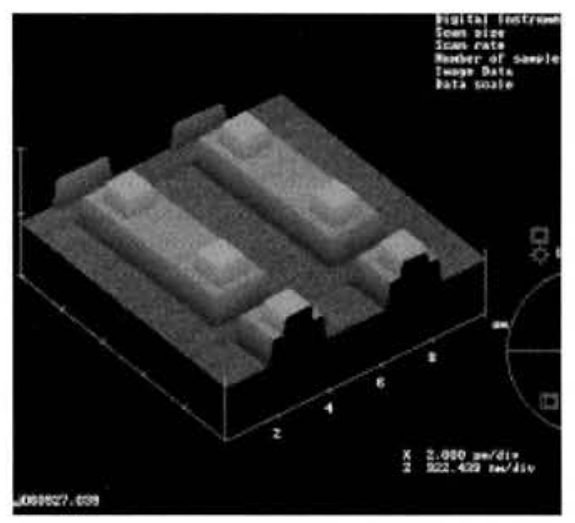

Figure 7. Dual damascene template from Toppan, Inc.

In the simplest permutation of this process, an inorganic dielectric is deposited exactly as it is in the photolithography process except that no etch stop is included in the layer. A sacrificial imprint resist is then patterned over the dielectric using the 
multilayer template. An example of images printed over dielectric using the Toppan template is provided as Figure 8.
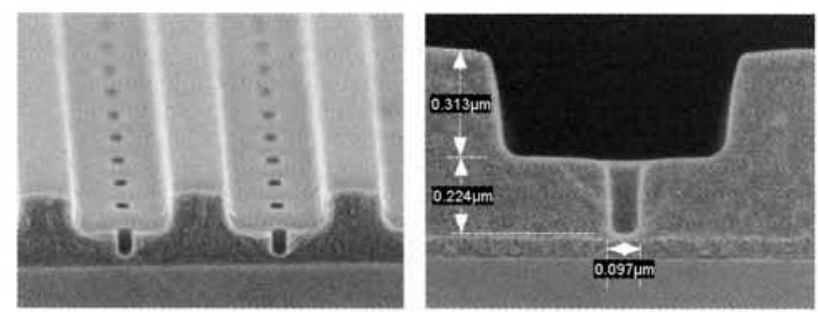

Figure 8. Scanning electon micrographs of imprints made over low 1 dielectric with a multilayer template. The nominal size of the vias is $100 \mathrm{~nm}$.

The resist pattern shown above can be transferred into the dielectric layer by a sequence of etch steps [18] that are all carried out in the same chamber as shown below in Figure 9. The resulting pattern in the dielectric is then ready for electroplating of copper and CMP to finish the wiring level.
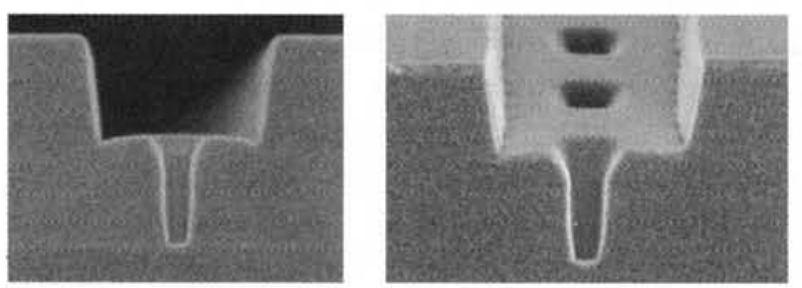

Figure 9. Low $\mathrm{k}$ dielectric pattened by etch transfer of imprinted images, nominal $100 \mathrm{~nm}$ vias

These structures were carried through metallization and CMP to produce via chains with differing lengths and via sizes. The electrical testing of these via chains revealed a $99.9968 \%$ yield for individual $100 \mathrm{~nm}$ vias and a yield of $96.83 \%$ for the chains with $1000,100 \mathrm{~nm}$ vias [18].

Two formal cost of ownership studies have been conducted on this process, one by Infineon [16] and one by SEMATECH [17]. Both found very substantial savings, greater than $20 \%$ reduction in wafer cost for products with 8 layers of metal. However, both assumed a thoughput of 20 wafers per hour, which has not yet been demonstrated.

\section{Conclusions:}

Step and Flash Imprint Lithography has made remarkable progress during the decade since it was first announced. Recent purchases of tools by SEMATECH and by semiconductor manufacturing firms will surely result in a rapid increase in the development of this technology and identification of any significant problems that have not emerged to date. Implementation of the technology below $22 \mathrm{~nm}$ will require improvements in alignment capability and full scale manufacturing will require higher throughput from tools. The issue of defect density still needs to be quantified, but the low cost of the technology and the very high resolution capability makes it a very attractive alternative for more defect tolerant applications including patterning of HDD media and advanced semiconductor memory applications.

\section{References}

1. M. Colburn, S. Johnson, S. Damle, T Bailey, B. Choi, M. Wedlake,; T. Michaelson, S.V. Sreenivasan, J. Ekerdt,. and C. G. Willson, Proc. SPIE, 3676 (1999) 379-389.

2. B. J. Choi, S. Johnson, M. Colburn, S.V. Sreenivasan, C. G. Willson, Journal of Int. Societies for Precision Engineering and Nanotechnology, 25(3), (2001) 192-199.

3. M. Colburn, B. J. Choi, S.V. Sreenivasan, R. Bonnecaze, C. G. Willson, Microelectronic Engineering, 75(3) (2004) 321-329.

4. S. Reddy, R. Bonnecaze, Microelectronics Engineering, 82, (2005) 60-70.

5. T. Bailey, B. J. Choi, M. Colburn, M. Meissl, S. Shaya; J. Ekerdt, S.V. Sreenivasan, C. G. Willson, J. Vac. Sci. Technol., B. 18(6) (2000) 3572-3577.

6. M. Lin, D. Hellebusch, K. Wu, E. K. Kim, Lu, H. Kuan; K. Liechti, J. Ekerdt, P. Ho, G. W. Willson, J. Micro/Nanolith. MEMS MOEMS, 7(3) (2008) 03300

7. F. Houle, et. al. Proc. SPIE, 6921, (2008) 69210B

8. I. McMackin, J. Maltabes, J. Perez, W. Martin, K. S. Selinidis, D. J. Resnick, S. V. Sreenivasan, Proc. SPIE, 7272 (2008)

9. S. Shiho, T. Hiraka, J. Mizuochi, Y. Nakanishi, S. Yusa, Y. Morikawa, H. Mohri, N. Hayashi Proc. SPIE, 7271 (2009)

10. I. Yoneda, S. Mikami, T. Ota, T. Koshiba, M. Ito, T. Nakasugi, T. Higashiki, Proc. SPIE, 6921 (2008)

11. S. J. Yeo, H. Kim; B. Eynon, Proc. SPIE, 6921 
(2008)

12. L. Litt, M. Malloy, Proc. SPIE, 7271 (2009)

13. M. Colburn, I. Suez, B.J. Choi, M. Meissl, T. Bailey, S.V. Sreenivasan, J. Ekerdt, and C. G. Willson, J. Vac. Sci. Technol., B, 19(6), 2685-2689 (2001)

14. S. Johnson, R. Burns, E. K. Kim, M. Dickey, G. Schmid, J. Meiring, S. Burns, C. G. Willson, J. Vac. Sci. Technol., B 23(6) (2005) 2553-2556

15. S. Postnikov, Infineon Technologies; presented at Semicon Europa 2007, Stuttgart, Germany

16. L. Litt, L.C., "Cost Analysis of Nanoimprint Lithography". Nanoimprint and Nanoprint Technology, Plenary Paper, Paris, France November 2006

17. D. Resnick, D. Mancini, S. V. Sreenivasan and C. G. Willson, Semiconductor International (June 1): (2002)

18. B. Chao; F. Palmieri, K. Jen; H. McMichael, C. G.Willson, J. Owens, R. Berger, K. Sotoodeh,
B. Wilks, J. Pham, R. Carpio, E. LaBelle, J. Wetzel, Proc SPIE, 6921 (2008)

19. C. Brooks, G. Schmid, M. Miller, S. Johnson, N. Khusnatdinov, D. LaBrake, D. Resnick, S. V. Sreenivasan, Proc. SPIE, 7271 (2009

20. R. Hershey, M. Miller, C. Jones, M. G. Subramanian, L. Xiaoming, G. Doyle, D. Lentz, D. LaBrake, Proc. SPIE, 6337 (2006): 63370M-1 - 63370M-10.

21. S. Johnson, D. Resnick, D. Mancini, K. Nordquist, W. Dauksher, K Gehoski, J. H. Baker, L. Baker, A, Dues, A. Hooper, T. Bailey, S. V. Sreenivasan, J. Ekerdt, C. G. Willson, Microelectronic Engineering, 67-68 (2003) 221-228.

22. G. Schmid, M. Stewart, J. Wetzel, F. Palmieri, J. Hao, Y. Nishimura, K. Jen, E. K. Kim, D. Resnick, A. Liddle, C. G. Willson, J. Vac. Sci. Technol., ST 24(3) (2006) 1283-1291. 